\title{
Cuba: Time to Expand Health in All Policies
}

\author{
Pastor Castell-Florit Serrate MD PhD DrSc and Pedro Más-Bermejo MD PhD DrSc
}

The Adelaide Statement on Health in All Policies (2010), lays out equity-based principles designed to guide policymakers on incorporating health and well-being components into the development, implementation and evaluation of policy and practice while moving towards shared governance at all levels-local, regional, national and international. Special emphasis is placed on cross-sector coordination to achieve policy goals, while improving health and well-being for all.[1]

In Cuba's case, experience in disaster preparedness, particularly for hurricanes, has shown good cross-sector coordination.[2] Zika serves as another recent example. First identified in Uganda in 1947, Zika, an emerging disease with outbreaks in Africa, Asia, the Pacific and the Americas and linked to neurological disorders in newborns, was declared a global health emergency by WHO on February 1, 2016.[3] In response, Cuba further stepped up measures for surveillance, prevention and control it had already announced in December 2015. Building on decades of experience fighting dengue, intensified efforts to stamp out Aedes aegypti and albopictus, the mosquitoes that transmit Zika (as well as dengue, chikungunya and yellow fever), and issued a national 11-point Zika Action Plan to prevent, detect and respond to these arboviral infections.

The Plan includes cross-sector policies, problem-solving and evaluation of actions in place to combat this wily, adaptable pest that thrives in urban and indoor settings. The epidemiologic picture is complicated by the fact that $80 \%$ of cases are asymptomatic, combined with environmental factors that include drought, which has led to improvised water storage. Establishing fixed fumigation schedules for residences and the public and private sectors; calling upon health, tourism, industry and civil defense authorities for input on policy and epidemiologic surveillance; and implementing a coordinated media campaign to inform the public about symptoms, fumigation protocols and vector control, are part of Cuba's national strategy.

Such coordination among government entities, civil society and the private sector is fundamental for interventions and programs to move towards health in all policies. The National Zika Action Plan has successfully prevented the disease from reaching the epidemic proportions seen in other countries in the Americas region, maintaining high levels of surveillance and public participation over time is a challenge. Still, the lessons learned from Zika-specifically how cross-sector collaboration, systematized national protocols, strict epidemiologic surveillance, strong regulatory frameworks and analysis of the effectiveness of these interventions for population health-are helpful for designing other strategies aimed at health in all policies.

Together, these tools can be applied to other health problems facing Cuba, including unintentional injuries, non-communicable diseases (especially cancer and cardiovascular diseases), and the health and social issues associated with a rapidly aging population. Complicated health concerns such as these require cross-sector coordination and synergy among programs and actions to achieve the greatest impact, as well as strong leader-
The Adelaide Statement on Health in All Policies outlines the need for a new social contract between all sectors to advance human development, sustainability and equity, as well as to improve health outcomes. This requires a new form of governance where there is joined-up leadership within governments, across all sectors and between levels of government. The Statement highlights the contribution of the health sector in resolving complex problems across government.

-Adelaide Statement on Health in All Policies, 2010, WHO

ship to implement policies that consciously integrate health and well-being.

One area where health in all policies is urgently needed for the important health benefits it provides, and due to the complexity of the issue, is ensuring food security. By implementing integrated, systematic policies across different sectors in the production, manufacture, marketing and distribution of food, Cuba can improve food security and foster healthier eating habits. In keeping with national policies already in place, which prioritize nutrition and sustainable food practices, strategies to strengthen alimentary safety-including defining the tasks and goals to achieve thisshould be coordinated more closely across different sectors and at all levels of government.

Systems and controls need to be in place and evaluated for health in all policies to be successful. This has proven challenging in Cuba, as it has in other countries as well. Nevertheless, the best results are being observed at the local level, where crosssector interventions and coordination are clear and unequivocal. Implementing health in all policies must consider all facets of daily life, which requires not only leadership from health authorities, but across all sectors of society and the economy. -1 -

1. World Health Organization, Government of South Australia. Adelaide statement on health in all policies: moving towards a shared governance for health and well-being [Internet]. Geneva: World Health Organization; 2010 [cited 2016 Mar 5]. 4 p. Available from: http://www.who.int/social_determinants/ hiap_statement_who_sa_final.pdf

2. Castell-Florit Serrate $\mathrm{P}, \bar{C}$ Carnota Lauzán O, Mirabal Jean-Claude M, Santana Espinosa MC, Cabrera González T, Hadad Hadad J, et al. Intersectorialidad en Cuba, su expresión a nivel global y local. Havana: Editorial Ciencias Médicas; 2008. Spanish.

3. World Health Organization. WHO statement on the first meeting of the IHR Emergency Committee on Zika virus and observed increase in neurological disorders and neonatal malformations [Internet]. Geneva: World Health Organization; 2016 Feb 1 [cited 2016 Mar 19]; [about 3 screens]. Available from: http://www.who.int/mediacentre/news/statements/2016/1st-emergency -committee-zika/en/

Submitted: March 17, 2016

Accepted for Publication: September 19, 2016

Disclosures: None

Correspondence: pmasbe@infomed.sld.cu 